\title{
Abordagem na sala de emergência: dotaçáo adequada de recursos de enfermagem
}

\author{
Emergency room approach: safe nursing staffing \\ Enfoque en la sala de urgencias: dotación adecuada de los recursos de enfermería
}

Maria Teresa Ferreira ${ }^{1}$
(D) https://orcid.org/0000-0003-1233-4676
Joaquim Filipe Fernandes ${ }^{2}$
(D) https://orcid.org/0000-0002-6043-1078
Rui Alberto Jesus ${ }^{2}$
(D) https://orcid.org/0000-0003-4224-1526
Isabel Maria Araújo ${ }^{2}$
(D) https://orcid.org/0000-0001-5143-4237

${ }^{1}$ Hospital Santa Maria Maior, E.P.E, Barcelos, Portugal

${ }^{2}$ Cooperativa de Ensino Superior Politécnico e Universitário, Famalicão, Portugal
Autor de correspondência:

Maria Teresa Ferreira

E-mail: teresa_ferr@hotmail.com

\section{Resumo}

Enquadramento: $\mathrm{O}$ prognóstico da pessoa em situação crítica na sala de emergência depende da eficácia da atuaçáo da equipa que a assiste.

Objetivos: Perceber e justificar a necessidade de alocar um enfermeiro que tenha como função a abordagem em exclusivo a clientes da sala de emergência num hospital da região de Lisboa e Vale do Tejo com um serviço de urgência polivalente.

Metodologia: Estudo retrospetivo, descritivo, exploratório, quantitativo. 3185 clientes críticos, recolha de informação por grelha de observação. Análise estatística foi realizada com o software IBM SPSS Statistics, versão 25.0. Recorreu-se ao teste de Kruskal-Wallis e Qui-quadrado.

Resultados: No ano de 2017, na sala de emergência admitiram-se em média 9 clientes por dia, os cuidados diretos prestados exigiram em média 45,4 minutos, 65\% foram admitidos na unidade de internamento médico-cirúrgico do serviço de urgência ou na área de observação clínica do mesmo serviço. Conclusáo: Emerge a necessidade de garantir dotação adequada aquando da prestação de cuidados à pessoa em situação crítica, sendo necessário um enfermeiro para funçôes exclusivas à sala de emergência.

Palavras-chave: emergência; sala de emergência; enfermagem; dotação segura

\section{Abstract}

Background: The prognosis of the critically ill person in the emergency room depends on the effective performance of the health team in operation.

Objectives: To understand and justify the need to allocate a nurse who works exclusively in the assistance to patients in the emergency room of a hospital in the Great Lisbon and Tagus Valley region with multipurpose emergency service.

Methodology: Retrospective, descriptive, exploratory, and quantitative study, with a sample of 3185 critically ill patients. Data collection through observation grid. Statistical analysis was performed using the IBM SPSS Statistics software, version 25.0. The Kruskal-Wallis test and chi-square test were used. Results: In 2017, an average of 9 patients per day were admitted in the emergency room. The direct care delivered lasted, on average, 45.4 minutes, $65 \%$ were admitted to the surgical admission unit service or the area of clinical observation of the emergency department.

Conclusion: It is crucial to ensure safe staffing in care delivery to the critically ill person, being necessary a nurse working exclusively in the emergency room.

Keyowrds: emergency; emergency room; nursing; safe staffing

\section{Resumen}

Marco contextual: El pronóstico de la persona en una situación crítica en la sala de urgencias depende de la eficacia del equipo que la asiste.

Objetivos: Comprender y justificar la necesidad de asignar un enfermero cuya función sea atender exclusivamente a los pacientes de la sala de urgencias de un hospital de la región de Lisboa y Valle del Tajo con un servicio de urgencias polivalente.

Metodología: Estudio retrospectivo, descriptivo, exploratorio y cuantitativo. 3185 pacientes críticos, recopilación de información mediante una parilla de observación. El análisis estadístico se realizó con el programa IBM SPSS Statistics, versión 25.0. Se utilizó la prueba de Kruskal-Wallis y la de ji al cuadrado. Resultados: En 2017, se admitió una media de 9 pacientes por día en la sala de urgencias, la atención directa prestada exigió un promedio de 45,4 minutos, el 65\% fue admitido en la unidad de internamiento médico-quirúrgico del servicio de urgencias o en el área de observación clínica del mismo servicio.

Conclusión: Es necesario garantizar una dotación adecuada cuando se atiende a la persona en una situación crítica, y es necesario un enfermero para las funciones exclusivas de la sala de urgencias.

Palabras clave: emergencia; sala de emergencia; enfermería; dotación segura

Como citar este artigo: Ferreira, M. T., Fernandes, J. F., Jesus, R. A., \& Araújo, I. M. (2020). Abordagem na sala de emergência: dotaçáo adequada de recursos de enfermagem. Revista de Enfermagem Referência, 5(1), e19086. doi: 10.12707/RIV19086 


\section{Introduçáo}

A especificidade dos cuidados numa sala de emergência é difícil de mensurar. Em Portugal, não existem números concretos de dotação segura para alocação dos enfermeiros à sala de emergência, nem normatização acerca da necessidade de exclusividade de funções em relação aos enfermeiros que aí prestam cuidados. Face ao número de admissóes diárias, ao tempo de permanência do cliente na sala de emergência, às exigências dos cuidados, e ao cansaço físico e psicológico que o exercício de funçóes em enfermagem acarreta pela prática clinica, entende-se que emerge a necessidade de alocar um enfermeiro a desempenhar funçóes exclusivas à sala de emergência.

Pela revisão bibliográfica compreende-se pelo Manual de Standards - Unidades de Urgência e Emergência (Direção-Geral da Saúde [DGS], 2016), que deve existir uma definição e atribuição de responsabilidades por cada posto de trabalho, como um instrumento de apoio indispensável para um melhor, e mais eficiente desenvolvimento das funçôes e atividades dos profissionais de saúde.

Deste modo, é importante perceber e justificar a necessidade de alocar um enfermeiro que tenha como função a abordagem em exclusivo a clientes da sala de emergência num hospital da regiáo de Lisboa e Vale do Tejo com um serviço de urgência polivalente.

\section{Enquadramento}

Os cuidados de saúde na área de urgência e emergência são um componente primordial à sociedade dos dias de hoje. Cada vez mais, há necessidade desses cuidados devido a acidentes, a violência urbana e situaçóes de doença relacionadas com o estilo de vida, o envelhecimento da população e co-morbilidades subsequentes.

De acordo com a DGS, o serviço de urgência (SU) existe para "tratar clientes em situaçôes urgentes e emergentes, oferecendo tratamento eficaz, eficiente e equitativo" (2001, p. 6-7). Para compreender a lógica de funcionamento do SU, a mesma entidade clarificou os conceitos de urgências como "todas as situaçôes clínicas de instalação súbita, desde as não graves até às graves, com risco de estabelecimento de falência de funçôes vitais" (DGS, 2001, p. 32) e emergências como "todas as situaçóes clínicas de estabelecimento súbito, em que existe, estabelecido ou eminente, o compromisso de uma ou mais funções vitais" (DGS, 2001, p. 32). Além disso, segundo o Manual de Recomendaçôes Técnicas para SU, publicado pela Administração Central do Sistema de Saúde (Administração Central do Sistema de Saúde, 2015), este tipo de serviço tem por objetivo a receção, diagnóstico e tratamento de clientes acidentados ou com doenças súbitas que necessitem de atendimento imediato em meio hospitalar. Assim, quem recorre a este tipo de serviço é atendido por uma equipa multidisciplinar com formação e competências específicas (Fernandes, Branco, \& Rodrigues, 2019). Segundo a DGS (2001), a sala de emergência tem como objetivo acolher doentes em alto risco de morte. Para Aehlert (2007), a prestação de cuidados, neste contexto, deve procurar a restauração rápida da viabilidade ventilatória e circulatória, com preservação dos órgãos vitais. Neste sentido, os cuidados de enfermagem a este tipo de doentes sáo altamente especializados, atentando principalmente à prevenção de complicações e minimização da incapacidade.

Após a estabilização do cliente na sala de emergência, o mesmo é transferido para o local previamente determinado, tendo em conta que a transferência deve ser segura e benéfica para o cliente. A responsabilidade da equipa que assiste a pessoa em situação crítica estende-se desde a admissão na sala de emergência até à sua saída, seja para um serviço dentro da unidade de saúde ou para outra unidade de saúde (Massada, 2002).

Num SU trabalham diversos profissionais de saúde, sendo que os enfermeiros constituem a maior porção das equipas. No entanto, atualmente, a escassez de recursos financeiros nas instituiçôes de prestação de cuidados de saúde traduz-se na falta destes profissionais, repercutindo-se na qualidade de atendimento prestado à população (Paixão, Campanharo, Lopes, Okuno, \& Batista, 2015). O SU, para além de ser o rosto e a porta de entrada de um hospital, pelas suas características próprias é um ambiente de stress, desprovido de padronização de cuidados, com imprevisibilidade e em que as açốes estão dependentes do tempo, com um ritmo de trabalho acelerado em que todos os minutos contam, onde se reflete a necessidade de haver uma equipa profissional muito bem preparada e capacitada com conhecimentos, com competências técnicas e psicomotoras, na tomada de decisão e rapidez na sistematização do trabalho em equipa.

A prestação de cuidados em situação crítica exige intervençôes complexas e atempadas, percorrendo a área extra-hospitalar, passando pelo transporte primário até à assistência intra-hospitalar (Ferreira et al., 2008).

Por forma a prestar cuidados aos clientes que são referenciados para o SU, surge a necessidade de dotar estas unidades de saúde com profissionais que concretizem este desígnio. Dotação surge como a "quantidade e tipo de pessoal necessário para a prestação de cuidados a clientes" (Hall, 2005, p. 11).

A dotação segura de recursos de enfermagem refere-se à correta dotação de enfermeiros em quantidade e qualidade, tendo em conta as necessidades dos clientes, sendo uma condição necessária para a qualidade dos cuidados prestados. De facto, é fundamental dispor de recursos adequados para que existam práticas seguras e que garantam o correto atendimento, segurança e satisfação da pessoa (Freitas \& Parreira, 2013), o que faz com que se questione se na realidade deste hospital se justifica a alocação de um enfermeiro para atendimento em exclusividade na sala de emergência.

A necessidade de adequar os recursos de enfermagem tendo em conta a segurança, o nível de necessidade de cuidados de enfermagem dos clientes, a qualidade dos cuidados de enfermagem, a carga de trabalho, o ambiente de trabalho e o nível de qualificação/experiência dos enfermeiros, fez emergir o conceito de dotaçâo segura em enfermagem (Freitas \& Parreira, 2013). A norma para o cálculo de dotaçóes seguras dos cuidados de enfermagem 
no SU utiliza a fórmula por posto de trabalho adaptado ao fluxo casuístico e fluxos de procura consoante a hora do dia, consoante o dia da semana e consoante o mês (Ordem dos Enfermeiros, 2014). Nesta fórmula, as horas de cuidados necessários têm por referência mínima o valor das unidades de cuidados intermédios, não havendo referência em específico ao trabalho de enfermagem na sala de emergência.

\section{Questão de Investigaçáo}

Quantas pessoas em situação crítica foram admitidas na sala de emergência num serviço de urgência polivalente, durante o ano 2017? Quanto tempo foi despendido por cada cliente e qual o motivo da admissão? Em que turno ocorreu maior afluência? Qual o destino do cliente?

\section{Metodologia}

A resposta a estas questóes teve como objetivo perceber a necessidade de alocar um enfermeiro para atendimento exclusivo a clientes da sala de emergência num hospital da regiáo de Lisboa e Vale do Tejo com um serviço de urgência polivalente. No contexto clínico onde foi realizado o estudo não existe diariamente um enfermeiro adstrito exclusivamente à sala de emergência, mas sim enfermeiros alocados a outros postos de trabalho e que têm por função, também, o apoio à sala de emergência. Realizou-se um estudo descritivo, exploratório, retrospetivo de natureza quantitativa, num serviço de urgência geral (SUG) de um hospital da área de Lisboa e Vale do Tejo categorizado pela Rede de Referenciação Hospitalar de Urgência/Emergência como serviço de urgência polivalente (SUP).

Englobou-se neste estudo, todos os clientes que deram entrada na sala de emergência desse hospital desde o dia 1 de Janeiro de 2017 até ao dia 31 de Dezembro, obtendo uma totalidade de 3185 clientes com informação completa ao nível dos registos consultados $(N=3185)$. Sáo admitidos na sala de emergência deste hospital clientes que entram diretamente do exterior (domicílio, via pública, etc.), alguns são admitidos de outras entidades hospitalares, e outros estáo à espera de observaçáo médica, ou já foram observados e, por algum motivo, o seu estado de saúde agudizou necessitando de cuidados que devem ser prestados nessa sala.

O SUG teve, em média, a afluência de 274 clientes por dia, durante o ano de 2017. Neste serviço, dividem-se os postos de trabalho por áreas de atendimento: a triagem, a sala de emergência, a área de ambulatório, a área de observação clínica, o serviço de urgência verdes e azuis (SUVA) e, ainda, a unidade de internamento médico-cirúrgico do SUG (UIMC). Existe, ainda, um enfermeiro responsável por informar e atender o utente/familiar do cliente que recorre a este $S U$ durante o período da manhã ou da tarde.

O rácio de enfermeiros a desempenhar funçóes de contacto direto com o cliente são 14 no turno da manhã, 14 no turno da tarde e 10 no turno da noite. Estão alocados à sala de emergência, para além do enfermeiro-chefe de equipa do turno, mais dois enfermeiros (consoante consideração do chefe de equipa de enfermagem) que são distribuídos, normalmente, na triagem, na área de ambulatório ou na área de observação clínica.

O grupo de enfermeiros responsáveis pela monitorização da sala de emergência possui uma base de dados, onde regista todas as admissóes realizadas nessa sala. Desta plataforma, foram consultadas e transcritas numa grelha de registos, as informaçóes relevantes para tentar responder às questōes de investigação deste estudo: número de admissões na sala de emergência, turno de ocorrência, o motivo de admissão, o tempo de permanência e o destino do cliente. Salienta-se que, o motivo de admissão do cliente na sala de emergência foi registado tendo por base os fluxogramas do Sistema de Triagem de Manchester, as vias verdes (quer referenciadas do exterior quer ativadas no SUG), as alterações eletrocardiográficas evidenciadas, e outros motivos mais específicos, como por exemplo, os clientes que vieram ao abrigo do programa da Urgência Metropolitana de Lisboa ou os que entram cadáveres. Esta grelha de registos foi construída pelos investigadores do estudo, fundamentada pelo modelo de registo institucional, tendo sido analisada e criticada por peritos na área de abordagem ao doente crítico.

Respeitaram-se procedimentos éticos: foi solicitada a autorizaçâo da Coordenadora Clínica e do enfermeiro-chefe do Serviço de Urgência Geral, bem como do Conselho de Administraçáo e, este estudo foi sujeito ao parecer da Comissão de Ética do hospital (Despacho do CA 16/10/2018). Importa realçar que os direitos dos clientes foram salvaguardados em todo o processo deste estudo, uma vez que não se usaram quaisquer elementos que permitissem identificar o cliente. Os dados foram organizados e a análise estatística foi realizada com o software IBM SPSS Statistics, versão 25.

Na caraterização global da amostra, e porque as variáveis numéricas têm distribuiçóes assimétricas, foram resumidas através da mediana, e percentis 25 e 75 , e são apresentadas no texto através das siglas $\mathrm{Md}, \mathrm{P}_{25}$ e $\mathrm{P}_{75}$, respetivamente. Para as variáveis qualitativas recorreu-se às frequências absolutas e relativas. Para verificar a existência de relaçôes entre as variáveis suprarreferidas, foram utilizados testes de Kruskal-Wallis (ex.: comparação do tempo de permanência do cliente na sala de emergência entre três grupos de sujeitos; ex.: admitidos nos turnos da manhã, tarde e noite) e testes do qui-quadrado (ex.: relação entre o turno e o motivo de admissão). Todos os valores de prova dos testes (valor- $p$ ) consideraram-se estatisticamente significativos se fossem inferiores a 0,05.

\section{Resultados}

Com o propósito de dar resposta ao objetivo do estudo apresentam-se os resultados descritivos e, posteriormente, a análise bivariada.

\section{Caracterização da amostra}


No ano de 2017, foram admitidos na sala de emergência do SUG 3185 clientes, sendo que 1705 (53,5\%) eram do sexo masculino e os restantes do sexo feminino. Admitindo que o ano de 2017 teve 365 dias, contabilizaram-se diariamente 8,7 admissóes. Os clientes admitidos na sala de emergência foram maioritariamente idosos, sendo a mediana de 74 anos de idade.

\section{Frequência de admissóes}

No que concerne à frequência de admissóes, na sala de emergência, por turno, verificou-se uma maior percentagem de admissôes no turno da manhã $(p<0,001)$, cerca de $40,4 \%$ e uma percentagem inferior no turno da noite, $25,5 \%$ (Tabela 1 ).

Tabela 1

Admissóes de clientes, por turno, na sala de emergência de um hospital da regiāo de Lisboa e Vale do Tejo durante o ano $2017(N=3185)$

\begin{tabular}{lcc}
\hline Turno & Número de admissóes & Média \\
\hline Manhã & 1286 & $40,4 \%$ \\
Tarde & 1087 & $34,1 \%$ \\
Noite & 812 & $25,5 \%$ \\
Total & 3185 & $100 \%$ \\
\hline
\end{tabular}

Foi ainda possível apurar que houve mais admissóes nos meses de outono e inverno (55,5\%), do que nos meses de primavera e verăo $(44,5 \%)$ com valor- $p<0,001$.

\section{Tempo de permanência}

A duração média dos 3185 episódios de emergência foi

de 45 minutos e 40 segundos, mas mais de metade destes foi mais curta (Moda $\left.=30^{\prime}\right)$.

Como se observa na Tabela 2, mais de quatro quintos dos sujeitos $(81,1 \%)$ ficaram $1 \mathrm{~h}$ ou menos na sala de emergência $(18,9 \%$ ficaram mais de $1 \mathrm{~h})$.

Tabela 2

Tempo de permanência na sala de emergência de um hospital da regiāo de Lisboa e Vale do Tejo durante o ano $2017(N=3185)$

\begin{tabular}{lcc}
\hline Tempo & Número de admissóes & Média \\
\hline Até 30 mins. & 1188 & $37,3 \%$ \\
31 a 60 mins. & 1394 & $43,8 \%$ \\
Mais de $1 \mathrm{~h}$ & 603 & $18,9 \%$ \\
Total & 3185 & $100 \%$ \\
\hline
\end{tabular}

Constatou-se (Tabela 3), que apesar de ser no turno da manhã que se admitiram mais clientes, a permanência dos clientes na sala de emergência durante o turno da manhã é significativamente menor (35 minutos) do que nos turnos da tarde e da noite ( 40 minutos; valor- $p=0,001$ no teste de Kruskal-Wallis).

Tabela 3

Tempo de permanência, por turno, dos clientes na sala de emergência de um hospital da regiäo de Lisboa e Vale do Tejo durante o ano de $2017(N=3185)$

\begin{tabular}{cccc}
\hline Turno & Número de admissōes & Média & Mediana \\
\hline Manhã & 1286 & 43,65 & 35,00 \\
Tarde & 1087 & 47,77 & 40,00 \\
Noite & 812 & 44,99 & 40,00 \\
Total & 3185 & 45,47 & 40,00 \\
\hline
\end{tabular}

Face à duração média de atendimento a cada cliente na sala de emergência e ao número de admissóes, constatou-se que, em cada turno, são necessárias entre 1,7h e 2,6h para o atendimento em exclusivo de clientes na sala de emergência (Tabela 4). 
Tabela 4

Duração do atendimento dos clientes na sala de emergência de um hospital da regiäo de Lisboa e Vale do Tejo durante o ano de $2017(N=3185)$

\begin{tabular}{lccc}
\hline Turno & $\begin{array}{c}\text { Duração média episódios de cada } \\
\text { admissão (mins.) }\end{array}$ & Número admissōes & $\begin{array}{c}\text { Duração total episódios de urgência } \\
\text { (h/dia) }\end{array}$ \\
\hline Manhã & 43,65 & 1286 & 2,6 \\
Tarde & 47,77 & 1087 & 2,4 \\
Noite & 44,99 & 812 & 1,7 \\
Total & 45,40 & 3185 & 6,6 \\
\hline
\end{tabular}

Motivo de admissão

Foi importante apurar o motivo de admissão dos clientes.

Assim, os dois maiores motivos pelos quais houve neces- sidade de ativação da sala de emergência foram: dispneia e alteraçóes eletrocárdiográficas (Tabela 5).

Tabela 5

Motivo de admissão dos clientes na sala de emergência de um hospital da região de Lisboa e Vale do Tejo durante o ano de $2017(N=3185)$

\begin{tabular}{|c|c|c|}
\hline Motivo de admissão & Número de admissōes & Média \\
\hline Dispneia & 699 & 21,9 \\
\hline Alterações eletrocardiográficas & 652 & 20,5 \\
\hline Via Verde AVC & 266 & 8,4 \\
\hline Alteração estado de consciência & 221 & 6,9 \\
\hline Convulsão & 168 & 5,3 \\
\hline Dor torácica & 156 & 4,9 \\
\hline Paragem cárdio-respiratória & 154 & 4,8 \\
\hline Via Verde Coronária & 137 & 4,3 \\
\hline Grande traumatismo & 128 & 4,0 \\
\hline Estado de inconsciência/Síncope & 84 & 2,6 \\
\hline Via Verde Sépsis & 70 & 2,2 \\
\hline Hemorragia intracraniana & 55 & 1,7 \\
\hline Hemorragia gastrointestinal & 53 & 1,7 \\
\hline Dor abdominal & 52 & 1,6 \\
\hline Palpitações & 42 & 1,3 \\
\hline Sobredosagem e envenenamento & 37 & 1,2 \\
\hline Outros & 211 & 6,7 \\
\hline Total & 3185 & 100,0 \\
\hline
\end{tabular}

Nota. AVC = acidente vascular cerebral.

Relativamente ao destino dos clientes, assistidos na sala de emergência, constata-se que a maior parte foram internados na UIMC ou encaminhados para a área de observação clínica (Tabela 6). 
Tabela 6

Destino dos clientes assistidos na sala de emergência de um hospital da regiäo de Lisboa e Vale do Tejo durante o ano de $2017(N=3185)$

\begin{tabular}{lcc}
\hline Destino & Número de admissóes & Média \\
\hline Unidade de Internamento Médico Cirúrgico & 1178 & 37,0 \\
Observaçáo Clínica & 893 & 28,0 \\
Unidade Cuidados Intensivos & 264 & 8,3 \\
Sala Hemodinâmica & 159 & 5,0 \\
Morgue & 146 & 4,6 \\
Ambulatório & 135 & 4,2 \\
Unidade Neurologia & 133 & 4,1 \\
Bloco Operatório & 86 & 2,7 \\
Outro hospital & 66 & 2,1 \\
Unidade Cuidados Coronários & 48 & 1,5 \\
Outros & 66 & 2,5 \\
Total & 3185 & 100,0 \\
\hline
\end{tabular}

\section{Discussão}

O estudo, aqui apresentado, abrangeu a análise dos 3185 clientes que foram admitidos na sala de emergência de um hospital da região de Lisboa e Vale do Tejo. Os clientes posicionavam-se, maioritariamente, na faixa etária superior a 60 anos. Este perfil de clientes é corroborado por diferentes estudos realizados em contexto de atendimento em situaçōes urgentes/emergentes (Barreira, Martins, Silva, Preto, \& Preto, 2019; Fernandes et al., 2019).

O estudo evidencia que, na maioria das admissóes, o cliente permanece na sala de emergência mais de quarenta e cinco minutos. Assim, diariamente são necessárias cerca de sete horas de cuidados, para a assistência direta ao cliente, prestados por pelo menos um enfermeiro. Salienta-se o facto de não estarem contemplados neste tempo, os cuidados indiretos, ou seja, o período de confirmação de todas as condições da sala de emergência, bem como a reposição de stocks e confirmaçôes/verificações dos materiais e equipamentos. Além do mais, faz parte das funçôes de enfermagem a manutenção da sala de emergência para usar prontamente e parte das atividades aí executadas são da competência dos enfermeiros e, mesmo as ações exclusivas dos médicos, requerem a colaboração dos enfermeiros.

Emergiu da análise dos dados que os principais motivos de admissão foram a dispneia e alteraçóes eletrocardiográficas, e que a maioria dos clientes atendidos na sala de emergência foram internados na UIMC ou ficaram na área de observação clínica. Foi possível apurar que a frequência de admissóes foi mais significativa no turno da manhã, comparado com o turno da tarde e da noite, e verificou-se que o tempo de permanência do cliente na sala de emergência foi inferior no turno da manhã. Como o propósito principal deste estudo foi perceber a necessidade de alocar um enfermeiro exclusivamente para atendimento a clientes da sala de emergência realça-seque, quer a UIMC quer a área de observaçáo clínica pertencem ao SUG. Assim, o cliente continua sob cuidados dos enfermeiros do SU. Como já referido, os enfermeiros que estão adstritos à sala de emergência, também, estão alocados a outros postos de trabalho. Se forem chamados à sala de emergência, a área de observação clínica, a triagem ou a área de ambulatório, ficam sob a responsabilidade de um único enfermeiro devido à ausência do colega que assiste a sala de emergência.

Massada (2002), explica que a sala de emergência deve estar sempre preparada para o início de uma emergência; o trabalho é feito em equipa, num espírito de coesão e linguagem comum, o papel de cada um está definido e as suas responsabilidades sob a coordenação de um elemento previamente conhecido. Na realidade descrita por Massada (2002), existem dois médicos e dois enfermeiros na sala de emergência, em presença física 24 horas por dia, sendo que um enfermeiro está adstrito exclusivamente à sala de emergência e outro à equipa de emergência intra-hospitalar e sempre que necessário, existe a possibilidade de reforço com um terceiro enfermeiro. Importa referir que as funçôes, bem como a formação destes enfermeiros, encontra-se criteriosamente definida.

Realça-se que, os enfermeiros que são ativados para sala de emergência aquando do accionamento do alarme sonoro, estavam anteriormente a prestar cuidados noutra área de atendimento, ficando essa área de atendimento com menos um enfermeiro enquanto este presta cuidados na sala de emergência. Contudo, por vezes, as admissóes na sala de emergência são simultâneas, tendo nessa altura a equipa de emergência necessidade de maior apoio, não sendo suficientes por vezes, três enfermeiros para dar assistência a dois clientes da sala de reanimação em simultâneo.

Os resultados aqui apresentados fundamentam a necessidade de alocar um enfermeiro para garantir dotaçóes 
seguras na sala de emergência, no contexto onde se desenvolveu o estudo. No entanto, vários estudos descrevem a sobrecarga de trabalho e o cansaço dos profissionais de saúde como ameaças à segurança do cliente e, nesse sentido, a The Joint Comission (2011) refere que um dos fatores para a ocorrência de eventos adversos na segurança do cliente é a fadiga dos profissionais de saúde devido à sobrecarga de trabalho, turnos longos, horas de trabalho acima das contratualizadas e insuficiência de dotação. De realçar que, o conceito de carga de trabalho envolve não só a dimensão biológica, mas também outras esferas envolvidas no trabalho, tais como, a mental e psicoafectiva. Foi corroborado o parecer solicitado à Ordem dos Enfermeiros (14/2018) acerca desta temática (Ordem dos Enfermeiros, 2018). A mesa do colégio da especialidade em enfermagem médico-cirúrgica, referencia que a necessidade de cuidados específicos emergentes não é padronizada e, portanto, não está sujeita a determinação de fluxos perfeitamente controláveis. Deste modo, a disponibilidade da equipa deve ser imediata, não podendo estar dependente da substituição noutros postos/áreas de trabalho.

A Circular Normativa 002/2018 publicada pela DGS (2018) reforça que, dada a complexidade dos clientes alocados à sala de emergência, a equipa aí adstrita deve ser detentora de formação especializada específica, da responsabilidade da instituição, de forma a responder com eficiência às situaçóes quer sejam de origem interna quer sejam ativadas através da triagem de prioridades.

Reconhecem-se as limitaçóes deste estudo. As mesmas estão relacionadas com a metodologia utilizada, com a escassez de registos dos cuidados prestados pelos enfermeiros (como por exemplo, o tempo necessário para operacionalizar a sala de emergência de forma a ficar apta para receber novo cliente) e investigação produzida para comparação de resultados. Realça-se a dificuldade em debater esta realidade com outra de um hospital em Portugal, uma vez que não foi encontrada nenhuma normatização acerca da exclusividade, ou não, de pessoal de enfermagem para a assistência à pessoa em situação crítica na sala de emergência. Assim, sugere-se a continuidade de estudos sobre esta temática, noutros contextos clínicos desta prática.

\section{Conclusão}

Os profissionais de saúde que trabalham no SUG do hospital onde se realizou o estudo, além de realizarem as suas atividades num clima de imprevisibilidade e incerteza, que requer conhecimento, concentração, rapidez de raciocínio e rapidez na realização do processo de tomada de decisão, ainda, são ativados para a prestação de cuidados na sala de emergência onde permanecem durante parte do turno, ficando a restante equipa sobrecarregada. A assistência de qualidade só é possível quando as instituiçóes promovem condiçóes de trabalho, com recursos físicos e humanos e processos institucionais coerentes para uma prática segura.

Face ao exposto e aos resultados obtidos, consideramos vantajoso e necessário para a excelência dos cuidados prestados, bem como, a satisfação de todos os envolvidos, a alocação de um enfermeiro para a prestação de cuidados em exclusivo na sala de emergência deste hospital.

Apesar da pesquisa bibliográfica realizada, não foi possível encontrar estudos em Portugal que atentem esta necessidade nem bibliografia acerca desta temática, emergindo assim, a necessidade de normalização para uma prática segura na dotação de enfermeiros na sala de emergência.

\section{Contribuiçáo de Autores}

Conceptualização: Ferreira, M. T.

Análise formal: Jesus, R. A.

Metodologia: Ferreira, M. T.

Administração do projeto: Ferreira, M. T.

Supervisão: Araújo, I. M.

Redação - rascunho original: Ferreira, M. T.

Redação - revisão e edição: Araújo, I. M., Fernandes, J. F.

\section{Referências bibliográficas}

Administração Central do Sistema de Saúde. (2015). Manual de recomendaçöes técnicas para serviços de urgências: RT 11/2015. Recuperado de http://www2.acss.min-saude.pt/Portals/0/Urg\%C3\%AAncias_\%20final.pdf

Aehlert, B. (2007). Emergência em cardiologia: Suporte avançado de vida em cardiologia. Recuperado de https://docero.com.br/ $\mathrm{doc} / \mathrm{n} 0 \mathrm{n} 81 \mathrm{~s} 0$

Barreira, I., Martins, M., Silva, N., Preto, P., \& Preto, L. (2019). Resultados da implementaçáo do protocolo da via verde do acidente vascular cerebral num hospital português. Revista de Enfermagem Referência, 4(22),117-126. doi: 10.12707/RIV18085

Direção-Geral da Saúde. (2018). Circular normativa 002/2018: Sistemas de triagem dos serviços de urgência e referenciação interna imediata. Recuperado de https://www.dgs.pt/directrizes-da-dgs/normas-e-circulares-normativas/norma-n-0022018-de-090120181.aspx

Direção-Geral da Saúde. (2016). Manual de standards: Unidades de urgência e emergência. Recuperado de https://www.dgs.pt/departamento-da-qualidade-na-saude/ficheiros-anexos/manual_de_standards_servicos-de-urgencia-e-emergencia_me-26-1_01_print.aspx

Direçấo-Geral da Saúde. (2001). Rede de referenciação hospitalar de urgêncialemergência. Recuperado de from https://www.dgs.pt/planeamento-de-saude/hospitais/redes-HYPERLINK "https://www.dgs. pt/planeamento-de-saude/hospitais/redes-referenciacao-hospitalar/ rede-de-referenciacao-hospitalar-de-"referenciacao-hospitalar/ rede-de-referenciacao-hospitalar-de-urgenciaemergencia.aspx

Fernandes, S. M., Branco, M. A., \& Rodrigues, P. A. (2019). A pessoa em situação crítica submetida a ventilação não invasiva num serviço de urgência. Revista de Enfermagem Referência, 4(22), 13-22. doi: 10.12707/RIV19027

Ferreira, F., Andrade, J., Mesquita, A., Campello, G., Dias, C., \& Granja, C. (2008). Sala de emergência: Análise e avaliação de um modelo orgânico funcional. Revista Portuguesa de Cardiologia, 27(7-8), 889-900.

Freitas, M. J., \& Parreira, P. M. (2013). Dotação segura para a prática de enfermagem: Operacionalidade do conceito e o seu impacto nos resultados. Revista de Enfermagem Referência, 3(10), 171178. doi: 10.12707/RIII12125

Hall, L. (2005). Quality work environments for nurse and patient 
safety. Toronto, Canadá: Jones and Bartlett Publishers. Recuperado de https:/ https://books.google.pt/books?id=nJu2fNDgFhYC\&pg=PP6\&lpg=PP6\&dq=Quality+work+en vironments+for+nurse+and+patient+safety.+Sudbury:+Jones+and + Bartlett $\&$ source $=$ bl\&ots $=S Z$ YW9FbJL1 \&sig $=$ AC fU3U2TuB-5J2gGdrR6iGxcRRTjrUH2hA\&hl=pt-PT\&sa=X\&ved=2ahUKEwjRnsj1zYvnAhUXDGMBHfgMDMYQ6AEwAnoECAYQAQ\#v=onepage\&q=Quality\%20work\%20 environments $\% 20$ for $\% 20$ nurse $\% 20$ and $\% 20$ patient $\% 20$ safety.\%20Sudbury\%3A\%20Jones\%20and\%20Bartlett \&f=false

Massada, R. (2002). Avaliação e ressuscitação do doente com trauma grave: Normas de orientaçáo clínica e administrativa. Porto, Portugal: Medisa. Recuperado de http://files.jvilelas.webnode. pt/200000147-5cb905db2e/Avalia\%C3\%A7\%C3\%A3o\%20 e\%20Ressuscita\%C3\%A7\%C3\%A3o\%20do\%20doente\%20 com\%20trauma.pdf
Ordem dos Enfermeiros. (2014). Norma para o cálculo de dotaçôes seguras dos cuidados de enfermagem. Recuperado de https://www. ordemenfermeiros.pt/media/8332/pontoquatro_norma_de_dotacoesseguras_dos_cuidados_de_enfermagem_ag_30_05_2014_ aprovado_por_maioria_proteg.pdf

Ordem dos Enfermeiros. (2018). Parecer emitido pela mesa do colégio da especialidade em enfermagem médico-cirúrgica 14/2018. Recuperado de https://www.ordemenfermeiros.pt/media/8287/parecern\%C2\%BA-14_2018_rectificado.pdf

Paixão, T. C., Campanharo, C. R., Lopes, M. C., Okuno, M. F., \& Batista, R.E. (2015). Dimensionamento de enfermagem em sala de emergência de um hospital-escola. Revista Escola de Enfermagem da Universidade de São Paulo, 49(3), 486-493. doi: 10.1590/ S0080-623420150000300017

The Joint Commission. (2011). Sentinel event alert. Recuperado de http://www.jointcommission.org/assets/1/18/SEA_48.pdf 